This is an electronic reprint of the original article. This reprint may differ from the original in pagination and typographic detail.

Author(s): Banichuk, Nikolay; Jeronen, Juha; Neittaanmäki, Pekka; Tuovinen, Tero

Title: $\quad$ Static instability analysis for travelling membranes and plates interacting with axially moving ideal fluid

Year: $\quad 2009$

Version:

Please cite the original version:

Banichuk, N., Jeronen, J., Neittaanmäki, P., \& Tuovinen, T. (2009). Static instability analysis for travelling membranes and plates interacting with axially moving ideal fluid. Journal of Fluids and Structures, 26(2), 274-291.

https://doi.org/10.1016/j.jfluidstructs.2009.09.006

All material supplied via JYX is protected by copyright and other intellectual property rights, and duplication or sale of all or part of any of the repository collections is not permitted, except that material may be duplicated by you for your research use or educational purposes in electronic or print form. You must obtain permission for any other use. Electronic or print copies may not be offered, whether for sale or otherwise to anyone who is not an authorised user. 


\title{
Statical Instability Analysis for Travelling Membranes and Plates Interacting with Axially Moving Ideal Fluid
}

\author{
N. Banichuk ${ }^{\mathrm{a}}$, J. Jeronen ${ }^{\mathrm{b}, *}$, \\ P. Neittaanmäki ${ }^{b}$ and T. Tuovinen ${ }^{b}$ \\ anstitute for Problems in Mechanics RAS, prosp. Vernadskogo 101, block 1, Moscow \\ 119526, Russian Federation \\ ${ }^{\mathrm{b}}$ Department of Mathematical Information Technology, Mattilanniemi 2 (Agora), 40100 \\ University of Jyväskylä, Finland
}

\begin{abstract}
The out-of-plane instability of a moving plate, travelling between two rollers with constant velocity, is studied, taking into account the mutual interaction between the buckled plate and the surrounding, axially flowing ideal fluid. Transverse displacement of the buckled plate (assumed cylindrical), is described by an integrodifferential equation that includes the centrifugal force, the aerodynamic reaction of the external medium, the vertical projection of membrane tension, and the bending force. The aerodynamic reaction is found analytically as a functional of the displacement.

To find the critical divergence velocity of the moving plate and its corresponding buckling mode, an eigenvalue problem and variational principle are derived. Plate divergence, both within a vacuum and when submerged in an external medium, is investigated with the application of analytical and numerical techniques.
\end{abstract}

Key words: instability analysis, buckling, ideal fluid, axially moving materials, axial flow, FSI, paper web

\footnotetext{
* Corresponding author. Tel.: +358 40760 7328; fax: +358 142602771.
}

Email address: juha. jeronen@jyu.fi (J. Jeronen). 


\section{Introduction}

From the viewpoint of papermaking, the inherent mechanical instability of axially moving continua is an important question. Travelling flexible strings, membranes, beams and plates are the most common models. An extensive amount of research has been conducted on their various aspects. See e.g. Archibald and Emslie (1958), Miranker (1960), Swope and Ames (1963), Simpson (1973), Chonan (1986), Wickert and Mote (1990), Shen et al. (1995), Wang (2003), Shin et al. (2005), Sygulski (2007), and

Kulachenko et al. (2007a,b).

It is known from experimental studies and some theoretical estimations (Pramila 1986) that mechanical instability in a travelling paper web can arise at some critical velocities, and that the instability may occur in dynamical, i.e., flutter or statical, i.e. divergence, forms. These critical velocities are of both theoretical and practical interest, as they set an upper limit for the running speed of a paper machine, and consequently on the rate of paper production that can be achieved.

Some previous investigations (see, e.g., Chang and Moretti 1991) show that for a moving paper web under certain conditions, the value of divergence speed $V_{0}^{\text {div }}$ is less than the value of flutter speed $V_{0}^{\mathrm{fl}}$, i.e., $V_{0}^{\mathrm{div}}<V_{0}^{\mathrm{fl}}$. Thus, the speed $V_{0}$ for reliable, stable movement of the paper web must satisfy the condition $V_{0}<V_{0}^{\text {div }}$.

An important factor that affects the instability of the axially moving continuum is the interaction between the elastic continuum and the surrounding medium. The results of a study by Pramila (1986) show that the critical velocities and eigenfrequencies obtained using the results of Mujumdar and Douglas (1976), which neglect the web-air interaction, may be up to $400 \%$ too high.

Different approaches have been used in literature for taking into account the fluid-structure interaction. For example, in Watanabe et al. (2002), two different methods of analysis were developed in order to clarify the phenomenon of paper flutter. One of these was a flutter simulation using a Navier-Stokes code, and the other method was based on a potential flow analysis of an oscillating thin airfoil. In Wu and Kaneko (2005), both linear and nonlinear analyses of sheet flutter caused by fluid-structure interaction in a narrow passage were developed.

The simplest approach toward the fluid-structure interaction is to assume potential flow (Niemi and Pramila 1986, Pramila 1987). To solve the external aerodynamic problem, and to find the reaction of the surrounding medium, the finite element method has been used (Niemi and Pramila 1986). Added-mass approaches are also common, usually in combination with a finite element solution (Pramila 1986, Pramila 1987, and for a modi- 
fied version with boundary layer theory, Frondelius et al. 2006).

It has been noted (Pramila 1986) that in the case without longitudinal transportation of the vibrating medium, comparison of experimental and theoretical results shows that predictions based on the potential flow theory are within about $10 \%$ of the measured results. According to the same study, the boundary layer is negligible even in the case with longitudinal transportation, and thus potential flow predictions should apply there also. However, in a recent paper, Frondelius et al. (2006) show that the boundary layer does have an effect, especially near the critical velocity of the vibrating medium. While the eigenfrequency predictions agree with those from potential flow theory, the divergence velocities are found to be significantly higher.

Nevertheless, from an academic research viewpoint, the potential flow problem is a standard reference case. It has been studied for axially moving materials in stationary air (e.g. Pramila 1986), and for stationary structures in axial flow (e.g. Eloy et al. 2007). In this study, we will combine these two cases, solving the problem for a travelling web subjected to axial flow. We will find the lowest critical velocity, and its corresponding buckling shape. To do this, we apply the Eulerian concept of elastic stability that was first used by him for buckling analysis of compressed elastic columns. As usual, we will concentrate on small deformations and use linear theory. We will further assume that the deformation is constant in the $y$ (width) direction. It should be noted that the cylindrical deformation assumption is an approximation, due to the localisation of deformation near the free edges that has been observed in axially moving paper webs.

With the limitations of potential flow and cylindrical deformation, we gain the possibility for an analytical solution of the aerodynamic problem. The main contribution of this study is an analytical functional representation for the reaction the fluid exerts on the buckled plate, when axial motion is accounted for both the plate and the fluid.

The form of the present problem shares some similarities with the problems of pipes conveying fluid, and stationary structures subjected to axial flow. Indeed, a similar functional analytical solution for the reaction of the fluid has been found for a stationary plate in axial flow by Kornecki et al. (1976). For the similarities between the two mentioned areas, see the review by Païdoussis (2008).

Also, the numerical methods used for such problems are readily applicable here. Eloy et al. (2007) and Huang (1995), for example, have studied the flutter of a cantilevered plate. In both of the studies, a Galerkin approach with the vacuum vibration modes as the basis was used. This is the approach we will also apply.

The study is laid out as follows. First we set up the aeroelastic prob- 


\section{Insert Figure 1 here}

Figure 1. Travelling plate, supported at $x=-\ell$ and $x=+\ell$, buckling when its velocity is $V_{0}=V_{0}^{\text {div }}$. The displacement $w$ of the plate is assumed to be cylindrical, i.e., constant in the $y$ direction (perpendicular to the page, not shown). At infinity, the fluid moves along the $x$ axis with the velocity $v_{\infty}$. The plate loses its stability at some critical divergence velocity $V_{0}^{\text {div }}$.

lem, and present the analytical solution of the aerodynamical part. We insert the result into the original elastic equation, obtaining an aeroelastic equation. We then formulate in variational form the eigenvalue problem of the critical divergence velocity $V_{0}^{\text {div }}$, and make a few observations on the vacuum case. In the last section, we present some numerical results based on the aeroelastic equation and the Fourier-Galerkin method, and compare the predicted divergence velocity to known results.

\section{Governing equations of elastic instability of moving plate interacting with surrounding ideal fluid}

Consider at first small transverse cylindrical deformations of a plate travelling with constant velocity $V_{0}$ and interacting with surrounding air, modelled as ideal fluid. The plate moves between rollers placed at $x=-\ell$ and $x=\ell$ along the $x$ axis. See Figure 1 .

We will work in the context of the flat panel model, which describes cylindrical deformations of a plate. Note that although the equations look similar, this model is not the same as the one-dimensional beam model. See Timoshenko and Woinowsky-Krieger (1959), and Bisplinghoff and Ashley (1962).

The corresponding equilibrium equation has the form

$$
\left(m V_{0}^{2}-T\right) \frac{\mathrm{d}^{2} w}{\mathrm{~d} x^{2}}+D \frac{\mathrm{d}^{4} w}{\mathrm{~d} x^{4}}=q_{f},
$$

where $m$ is the areal mass density $\left([m]=\mathrm{kg} / \mathrm{m}^{2}\right)$ of the plate, $T$ is the tension per unit length in the $x$ direction, $D$ is the cylindrical rigidity of the plate, $q_{f}$ is the aerodynamic reaction (expressed as a volume force, $\left[q_{f}\right]=$

$\mathrm{N} / \mathrm{m}^{3}$ ) and $w$ is the small displacement in the $z$ direction (see Figure 1 ). The cylindrical rigidity is given by (Timoshenko and Woinowsky-Krieger 1959, p. 5)

$$
D=\frac{E h^{3}}{12\left(1-v^{2}\right)},
$$

where $E$ is the Young modulus, $h$ is the plate thickness, and $v$ is the Poisson ratio of the plate. The cylindrical rigidity is also known as the flexural rigidity. 
We will work in a dimensionless notation, where we define

$$
\begin{gathered}
x^{\prime} \equiv x / \ell \quad \text { and } \\
w^{\prime}\left(x^{\prime}\right) \equiv w\left(\ell x^{\prime}\right) .
\end{gathered}
$$

For brevity of notation, the primes and the $\ell$ s will be omitted during derivation, and the final results will be given with the $\ell$ s re-inserted.

We suppose here that the plate is simply supported at both ends. The corresponding boundary conditions at $x=-1$ and $x=+1$ (where $x$ is now dimensionless) are given as

$$
(w)_{x=-1}=\left(\frac{\mathrm{d}^{2} w}{\mathrm{~d} x^{2}}\right)_{x=-1}=0, \quad(w)_{x=1}=\left(\frac{\mathrm{d}^{2} w}{\mathrm{~d} x^{2}}\right)_{x=1}=0 .
$$

The divergence phenomenon consists of appearance of nontrivial $(w \not \equiv$ 0 ) equilibrium forms of the plate at some values of velocity $V_{0}$. To derive the statical equation of equilibrium for determining the buckled forms of the plate and the critical divergence speed $V_{0}^{\text {div }}$, it is necessary to derive the expression for aerodynamic reaction $q_{f}$ as a function of $w$.

Considering the cylindrical deformation assumption, we shall limit our study to a solenoidal flow in two dimensions. We consider the $x z$ plane with Cartesian coordinates, setting the $x$ axis parallel to the undisturbed flow of the fluid and the movement of the plate.

The aerodynamic velocity potential of airflow with respect to the moving plate surface, and total pressure, have the forms

$$
\begin{gathered}
\Phi(x, z)=x \cdot\left(v_{\infty}-V_{0}\right)+\varphi(x, z) \\
P(x, z, t)=p_{\infty}+p(x, z, t) .
\end{gathered}
$$

Here $v_{\infty}$ and $p_{\infty}$ are respectively the given velocity and pressure of the fluid at infinity, and $\varphi$ and $p$ are aerodynamic disturbances of the velocity potential and pressure.

The total reaction force $q_{f}$ exerted by the fluid is equal to the difference of pressure between the upper and lower faces of the plate,

$$
\begin{aligned}
q_{f}(x) & =P^{-}(x)-P^{+}(x) \\
& =p^{-}(x)-p^{+}(x), \quad-1 \leq x \leq 1
\end{aligned}
$$

where the superscript notation is defined for any function $f$ as

$$
f^{ \pm}(x) \equiv \lim _{z \rightarrow 0^{ \pm}} f(x, z) .
$$


The upper (lower) signs correspond to each other.

To derive an expression for the pressure $p(x, z)$, we use Bernoulli's integral (see e.g. Sedov 1972, pp. 155-157):

$$
\frac{1}{2}(\nabla \Phi)^{2}+\frac{1}{\rho_{f}} p=C ，
$$

where $\rho_{f}$ is the density of the fluid and $C$ is a constant. The pressure $p(x, z)$ thus depends on the velocity potential $\Phi(x, z)$.

The potential $\Phi=\Phi(x, z)$ can be found by solving a Neumann boundary value problem for the region exterior to the plate. Instead of $\Phi$, we shall consider the disturbance potential function $\varphi=\varphi(x, z)$ related to $\Phi$ by the formula (6). Regarding $\varphi$ and $w$ and their first derivatives as small, we linearise the aerodynamic problem and introduce a boundary condition that the fluid does not cross the surface of the plate, relating this surface to the boundary of the cut $z=0,-1 \leq x \leq 1$.

By inserting (6) into (9) and omitting the second-order small terms $(\partial \varphi / \partial x)^{2}$ and $(\partial \varphi / \partial z)^{2}$, we obtain an expression for the pressure $p$. Inserting the result into (8), we have

$$
q_{f}=p^{-}-p^{+}=\rho_{f} \cdot\left(v_{\infty}-V_{0}\right)\left[\left(\frac{\partial \varphi}{\partial x}\right)^{+}-\left(\frac{\partial \varphi}{\partial x}\right)^{-}\right] .
$$

The boundary condition that the fluid does not cross the surface of the plate, $v_{n}=(\bar{n} \cdot \bar{v})=(\bar{n} \cdot \nabla \Phi)=0$, is represented in the following linearised form,

$$
v_{n}=\left(v_{\infty}-V_{0}\right) n_{x}+\frac{\partial \varphi}{\partial z} n_{z}=0,
$$

where $n_{x}=-\mathrm{d} w / \mathrm{d} x$ and $n_{z}=1$ are the projections of the unit vector $\bar{n}$ that is normal to the plate surface. Consequently, we have

$$
\frac{\partial \varphi}{\partial z}=\left(v_{\infty}-V_{0}\right) \frac{\mathrm{d} w}{\mathrm{~d} x} .
$$

Thus the linearised aerodynamic problem can be written as

$$
\begin{gathered}
\triangle \varphi \equiv \frac{\partial^{2} \varphi}{\partial x^{2}}+\frac{\partial^{2} \varphi}{\partial z^{2}}=0 \\
\left(\frac{\partial \varphi}{\partial z}\right)^{ \pm}=\left(v_{\infty}-V_{0}\right) \frac{\mathrm{d} w}{\mathrm{~d} x}, \quad z=0,-1 \leq x \leq 1 \\
(\nabla \varphi)_{\infty}=0 .
\end{gathered}
$$


The domain of the aerodynamic problem is infinite. It consists of the whole $x z$ plane with the exception of the cut at $z=0,-1 \leq x \leq 1$, which is our linearised representation of the space occupied by the plate (note boundary condition (14)). Although we consider an axially moving plate, for the purposes of our analysis the plate only exists on the interval $-1 \leq$ $x \leq 1$.

The solution of the boundary value problem (13)-(15) and the derivation of the formula for the aerodynamic force has been presented in Banichuk and Neittaanmäki (2008) (see also Appendix A) as a function of the plate transverse displacement $w$ in the following form:

$$
\begin{aligned}
q_{f} & =\rho_{f} \cdot\left(v_{\infty}-V_{0}\right)\left[\left(\frac{\partial \varphi}{\partial x}\right)^{+}-\left(\frac{\partial \varphi}{\partial x}\right)^{-}\right] \\
& =-\rho_{f} \cdot\left(v_{\infty}-V_{0}\right)^{2} \frac{\partial}{\partial x} \int_{-1}^{1} N(\xi, x) \frac{\mathrm{d} w}{\mathrm{~d} x}(\xi) \mathrm{d} \xi,
\end{aligned}
$$

where the aerodynamic kernel $N$ is defined as

$$
N(\xi, x)=\frac{1}{\pi} \ln \left|\frac{1+\Lambda}{1-\Lambda}\right|
$$

and

$$
\Lambda(\xi, x)=\left[\frac{(1-x)(1+\xi)}{(1-\xi)(1+x)}\right]^{1 / 2} .
$$

One needs to be careful with the derivative on the left side of the integral in $q_{f}$ because the aerodynamic kernel $N(\xi, x)$ is singular. It can be shown (for details, see Banichuk et al. 2008) that the $L_{1}$ norm of $N$ is finite, but that of $\partial N / \partial x$ is not, so we cannot directly take the derivative operator into the integral. However, because the integral in $q_{f}$,

$$
\int_{-1}^{1} N(\xi, x) \frac{\mathrm{d} w}{\mathrm{~d} x}(\xi) \mathrm{d} \xi \equiv F(x),
$$

is absolutely convergent, the function $F(x)$ is bounded. Thus, two different approaches may be employed. If the integral is evaluated first, the resulting function can be differentiated by all the usual methods. However, because the antiderivative is not known analytically, it may be easier in a numerical solution to use a weak form and integration by parts, as we do below. Integration by parts in the weak form is legitimate because the integrand of the weak form is a product of two bounded, integrable functions: the test function and $q_{f}=-\rho_{f}\left(v_{\infty}-V_{0}\right)^{2} \frac{\partial}{\partial x} F(x)$.

In summary, in this section we derived the following integro-differential 
equation for the stationary displacement function $w=w(x)$,

$$
\begin{aligned}
\left(m V_{0}^{2}-T\right) \frac{\mathrm{d}^{2} w}{\mathrm{~d} x^{2}}+D \frac{\mathrm{d}^{4} w}{\mathrm{~d} x^{4}} & = \\
& \quad \rho_{f} \cdot\left(v_{\infty}-V_{0}\right)^{2} \frac{\partial}{\partial x} \int_{-1}^{1} N(\xi, x) \frac{\mathrm{d} w}{\mathrm{~d} x}(\xi) \mathrm{d} \xi,
\end{aligned}
$$

which is considered with the corresponding boundary conditions (5). The equation is written in the dimensionless notation introduced above. Accounting for the span length $\ell$, equation (19) becomes

$$
\begin{aligned}
\left(m V_{0}^{2}-T\right) \frac{1}{\ell^{2}} \frac{\mathrm{d}^{2} w}{\mathrm{~d} x^{2}}+ & \frac{D}{\ell^{4}} \frac{\mathrm{d}^{4} w}{\mathrm{~d} x^{4}}= \\
& -\frac{1}{\ell} \rho_{f} \cdot\left(v_{\infty}-V_{0}\right)^{2} \frac{\partial}{\partial x} \int_{-1}^{1} N(\xi, x) \frac{\mathrm{d} w}{\mathrm{~d} x}(\xi) \mathrm{d} \xi,
\end{aligned}
$$

where $x$ and $w$ still represent the dimensionless quantities (3) and (4).

Equation (20), together with the boundary conditions (5), constitutes our eigenvalue problem. The minimal value of the parameter $V_{0}$ is called the critical divergence speed, if there exists a nontrivial function $w(x)$ satisfying equation (20) with boundary conditions (5). This function, if it exists, is known as the divergence shape or mode.

\section{Eigenvalue problem and variational principle}

Let us multiply the left and right hand sides of (19) by an arbitrary function $\phi(x)$ satisfying the boundary conditions (5). Then we integrate both sides with respect to $x$ over the interval $[-1,1]$ :

$$
\left(m V_{0}^{2}-T\right) \int_{-1}^{1} \frac{\mathrm{d}^{2} w}{\mathrm{~d} x^{2}} \phi \mathrm{d} x+D \int_{-1}^{1} \frac{\mathrm{d}^{4} w}{\mathrm{~d} x^{4}} \phi \mathrm{d} x=\int_{-1}^{1} q_{f} \phi \mathrm{d} x
$$

Performing integration by parts twice in the bending term and once in the other terms, we obtain

$$
\begin{gathered}
\left(T-m V_{0}^{2}\right) \int_{-1}^{1}\left(\frac{\mathrm{d} w}{\mathrm{~d} x}\right)\left(\frac{\mathrm{d} \phi}{\mathrm{d} x}\right) \mathrm{d} x+D \int_{-1}^{1}\left(\frac{\mathrm{d}^{2} w}{\mathrm{~d} x^{2}}\right)\left(\frac{\mathrm{d}^{2} \phi}{\mathrm{d} x^{2}}\right) \mathrm{d} x \\
-\rho_{f} \cdot\left(v_{\infty}-V_{0}\right)^{2} \int_{-1}^{1} \int_{-1}^{1} \frac{\mathrm{d} w}{\mathrm{~d} x}(\xi) N(\xi, x) \frac{\mathrm{d} \phi}{\mathrm{d} x}(x) \mathrm{d} \xi \mathrm{d} x=0 .
\end{gathered}
$$

Note that as discussed above, we have transferred the outer derivative in $q_{f}$ onto the $\phi$ we introduced in (21). Again accounting for an arbitrary length 
$\ell$, we have

$$
\begin{array}{r}
\left(T-m V_{0}^{2}\right) \frac{1}{\ell} \int_{-1}^{1}\left(\frac{\mathrm{d} w}{\mathrm{~d} x}\right)\left(\frac{\mathrm{d} \phi}{\mathrm{d} x}\right) \mathrm{d} x+\frac{D}{\ell^{3}} \int_{-1}^{1}\left(\frac{\mathrm{d}^{2} w}{\mathrm{~d} x^{2}}\right)\left(\frac{\mathrm{d}^{2} \phi}{\mathrm{d} x^{2}}\right) \mathrm{d} x \\
-\rho_{f} \cdot\left(v_{\infty}-V_{0}\right)^{2} \int_{-1}^{1} \int_{-1}^{1} \frac{\mathrm{d} w}{\mathrm{~d} x}(\xi) N(\xi, x) \frac{\mathrm{d} \phi}{\mathrm{d} x}(x) \mathrm{d} \xi \mathrm{d} x=0 .
\end{array}
$$

Rearranging the terms, we obtain

$$
\begin{aligned}
Q(w, \phi) \equiv-\left(m J_{C}+\rho_{f} J_{F}\right) V_{0}^{2}+\left(2 v_{\infty} \rho_{F} J_{F}\right) V_{0} \\
+\left(T J_{T}+D J_{B}-\rho_{F} v_{\infty}^{2} J_{F}\right)=0,
\end{aligned}
$$

where the functionals corresponding to the tension, bending, aerodynamic $(\mathrm{F})$, and centrifugal terms are

$$
\begin{aligned}
& J_{T}(w, \phi)=J_{C}(w, \phi)=\int_{-1}^{1}\left(\frac{\mathrm{d} w}{\mathrm{~d} x}\right)\left(\frac{\mathrm{d} \phi}{\mathrm{d} x}\right) \mathrm{d} x \\
& J_{B}(w, \phi)=\int_{-1}^{1}\left(\frac{\mathrm{d}^{2} w}{\mathrm{~d} x^{2}}\right)\left(\frac{\mathrm{d}^{2} \phi}{\mathrm{d} x^{2}}\right) \mathrm{d} x \\
& J_{F}(w, \phi)=\int_{-1}^{1} \int_{-1}^{1} \frac{\mathrm{d} w}{\mathrm{~d} x}(\xi) N(\xi, x) \frac{\mathrm{d} \phi}{\mathrm{d} x}(x) \mathrm{d} \xi \mathrm{d} x .
\end{aligned}
$$

We can now state the variational form of our eigenvalue problem: find the smallest positive $V_{0}$, and the corresponding divergence shape $w(x)$, such that equation (24) holds for all functions $\phi(x)$ that satisfy the boundary conditions (5). This is the weak form of the problem introduced in the previous section, and the same nomenclature applies.

\section{Plate divergence in vacuum}

If the motion of the plate is considered in a vacuum, then $\rho_{f}=0$ and consequently $q_{f}=0$. In this case, (19) becomes

$$
\left(m V_{0}^{2}-T\right) \frac{\mathrm{d}^{2} w}{\mathrm{~d} x^{2}}+D \frac{\mathrm{d}^{4} w}{\mathrm{~d} x^{4}}=0 .
$$

Because (26) contains only second and fourth derivatives, we can introduce an auxiliary function

$$
u \equiv \frac{\mathrm{d}^{2} w}{\mathrm{~d} x^{2}}
$$


describing the curvature of the plate, and formulate the corresponding eigenvalue problem for it:

$$
\begin{array}{cc}
\frac{\mathrm{d}^{2} u}{\mathrm{~d} x^{2}}+\lambda u=0, & -1 \leq x \leq 1 \\
u(-1)=0, & u(1)=0 .
\end{array}
$$

The parameter $\lambda$ in (28),

$$
\lambda=\frac{m V_{0}^{2}-T}{D},
$$

plays the role of the eigenvalue. The nontrivial solutions, i.e., eigenfunctions, of the eigenvalue problem (28)-(29) are determined as

$$
u(x)=A \sin \left(\sqrt{\lambda} \frac{x+1}{2}\right)+B \cos \left(\sqrt{\lambda} \frac{x+1}{2}\right),
$$

with two unknown coefficients $A$ and $B$ and an unknown eigenvalue $\lambda$. Applying the boundary conditions (29), we obtain

$$
\begin{gathered}
u(x)=A \sin \left(j \pi \frac{x+1}{2}\right) \\
\lambda=j^{2} \pi^{2}, \quad j=1,2, \ldots
\end{gathered}
$$

where $A \neq 0$ is an arbitrary constant. In divergence analysis made in the frame of the Eulerian concept of statical instability, the amplitudes of the eigenfunctions are unknown. Integrating (31) twice and accounting for the boundary conditions (5), we have for the displacement of the $j$ th mode the function

$$
w(x)=C \sin \left(j \pi \frac{x+1}{2}\right), \quad-1 \leq x \leq 1,
$$

where $C=-(2 / j \pi)^{2} A$ is an arbitrary constant. We have from (30) and (32) that the corresponding divergence speed is

$$
\left(V_{0 \text { vac }}^{\text {div } j}\right)^{2}=\frac{T+j^{2} \pi^{2} D}{m} .
$$

Thus, we observe that the shape of the eigenmode coincides with the membrane $(D=0)$ eigenmode regardless of the value of the bending rigidity $D$, but the bending rigidity contributes an additional term to the divergence speed.

Only the critical divergence speed has physical significance. It corresponds to the minimal eigenvalue, i.e., $j=1$. Inserting the omitted $\ell$ s, we have for the critical divergence speed

$$
\left(V_{0 \text { vac }}^{\text {div }}\right)^{2}=\frac{\pi^{2} D}{m \ell^{2}}+\frac{T}{m} .
$$


In the theory of paper web dynamics, the bending rigidity $D$ is a small parameter. If $D$ tends to zero, then the divergence speed tends to the limit value,

$$
V_{0 \text { vac }}^{\text {div }} \rightarrow \sqrt{\frac{T}{m}}=V_{0 \text { mem vac }}^{\text {div }}
$$

which corresponds to the critical divergence speed of a moving membrane (see, e.g., Chang and Moretti 1991).

We also observe by letting $D \rightarrow 0$ in (34) that for a membrane in vacuum, there is only one (degenerate) eigenvalue, and thus all displacement modes (33) correspond to the critical divergence speed. Because (33) is an infinite Fourier basis, this implies that a membrane in vacuum may take any shape as it approaches divergence. This is in contrast with the case with surrounding fluid, and also that of a plate in a vacuum, for both of which the divergence shape is unique.

\section{Numerical estimations}

In this section we consider the eigenvalue problem of equation (19) with the boundary conditions (5), applying numerical techniques in order to obtain concrete values for the critical divergence speed and the corresponding eigenmode of divergence.

The original problem can be approximated with a finite-dimensional problem in a standard form. We represent $w(x)$ as a Galerkin series in a Fourier type basis,

$$
w(x)=\sum_{n=1}^{n_{0}} f_{n} \Psi_{n}(x),
$$

where $n_{0}$ is a discretisation parameter and

$$
\Psi_{n}(x) \equiv \sin \left(n \pi \frac{x+1}{2}\right), \quad x \in[-1,1]
$$

are the (normalised) eigenmodes of free vibrations of a membrane in a vacuum. The basis satisfies the boundary conditions (5) by its construction.

By multiplying both sides of (19) with $\Psi_{j}$ (where $j=1,2, \ldots, n_{0}$ ), integrating over the domain $[-1,1]$ and inserting (37), we have, after rearranging the terms, the weak form

$$
\begin{aligned}
\left(T S_{j n}+D K_{j n}-\rho_{f} v_{\infty}^{2}\right. & \left.N_{j n}\right) f_{n}= \\
& \quad\left[\left(m S_{j n}+\rho_{f} N_{j n}\right) V_{0}^{2}-2 \rho_{f} v_{\infty} N_{j n} V_{0}\right] f_{n},
\end{aligned}
$$

where the summation convention has been used. For an arbitrary length $\ell$, 
equation (39) becomes

$$
\begin{aligned}
\left(\frac{T}{\ell} S_{j n}+\frac{D}{\ell^{3}} K_{j n}-\rho_{f} v_{\infty}^{2} N_{j n}\right) f_{n} & = \\
& {\left[\left(\frac{m}{\ell} S_{j n}+\rho_{f} N_{j n}\right) V_{0}^{2}-2 \rho_{f} v_{\infty} N_{j n} V_{0}\right] f_{n} . }
\end{aligned}
$$

The matrices $S_{j n}, K_{j n}$ and $N_{j n}$ are the discrete representations of the functionals $J_{C}=J_{T}, J_{B}$ and $J_{F}$ respectively, and are defined as

$$
\begin{aligned}
S_{j n} & \equiv \int_{-1}^{1} \frac{\mathrm{d} \Psi_{j}}{\mathrm{~d} x} \frac{\mathrm{d} \Psi_{n}}{\mathrm{~d} x} \mathrm{~d} x=\left(\frac{j \pi}{2}\right)^{2} \delta_{j n} \\
K_{j n} & \equiv \int_{-1}^{1} \frac{\mathrm{d}^{2} \Psi_{j}}{\mathrm{~d} x^{2}} \frac{\mathrm{d}^{2} \Psi_{n}}{\mathrm{~d} x^{2}} \mathrm{~d} x=\left(\frac{j \pi}{2}\right)^{4} \delta_{j n} \\
N_{j n} & \equiv \int_{-1}^{1} \int_{-1}^{1} \frac{\mathrm{d} \Psi_{n}}{\mathrm{~d} x}(\xi) N(\xi, x) \frac{\mathrm{d} \Psi_{j}}{\mathrm{~d} x}(x) \mathrm{d} \xi \mathrm{d} x,
\end{aligned}
$$

where $\delta_{j n}$ is the Kronecker delta. The expressions for $S_{j n}$ and $K_{j n}$ can be obtained by a direct analytical calculation of the antiderivative, the result of which is given above. The matrix $N_{j n}$ must be evaluated numerically because no closed-form solution for the antiderivative is available.

We see that the matrices $S_{j n}$ and $K_{j n}$ are diagonal. This is to be expected, because we work in a vacuum eigenmode basis, and these matrices correspond to physical effects already present in the vacuum case. However, the matrix $N_{j n}$ introduced due to the fluid-structure interaction is found to be diagonally dominated, but it is clearly not diagonal. Thus, when the plate is submerged in an external medium, couplings exist between the vacuum eigenmodes that cannot be reproduced by an added mass approach.

Writing (40) in matrix form, multiplying both sides by $\ell$, dividing by tension $T$ and applying (36), we obtain

$$
\begin{aligned}
& \left(S+\frac{D}{\ell^{2} T} K-\frac{\ell \rho_{f}}{m} \frac{v_{\infty}^{2}}{\left(V_{0}^{\mathrm{dmv}}\right)^{2}} N\right) f= \\
& \quad\left[\left(\frac{1}{\left(V_{0}^{\mathrm{dmv}}\right)^{2}} S+\frac{\ell \rho_{f}}{m} \frac{1}{\left(V_{0}^{\mathrm{dmv}}\right)^{2}} N\right) V_{0}^{2}-2 \frac{\ell \rho_{f}}{m} \frac{v_{\infty}}{V_{0}^{\mathrm{dmv}}} N \frac{V_{0}}{V_{0}^{\mathrm{dmv}}}\right] f,
\end{aligned}
$$

where we have abbreviated $V_{0}^{\mathrm{dmv}} \equiv V_{0 \text { mem vac }}^{\mathrm{div}}$. To simplify the notation, we define the dimensionless quantities 


$$
\begin{aligned}
\lambda & \equiv V_{0} / V_{0 \text { mem vac }}^{\text {div }} \\
\beta & \equiv \frac{D}{\ell^{2} T} \\
\gamma & \equiv \frac{\ell \rho_{f}}{m} \\
\theta & \equiv v_{\infty} / V_{0 \text { mem vac }}^{\text {div }} .
\end{aligned}
$$

Our eigenvalue problem thus becomes

$$
\left(S+\beta K-\gamma \theta^{2} N\right) f=\left[(S+\gamma N) \lambda^{2}-2 \gamma \theta N \lambda\right] f .
$$

Equation (47) is a quadratic eigenvalue problem, which can be reduced to a (twice larger) standard linear generalised eigenvalue problem by applying one of the companion linearisations (see, e.g., the extensive review article Tisseur and Meerbergen 2001, p. 253). The problem has the dimensionless eigenvalue $\lambda$ and three dimensionless parameters, $\beta, \gamma$ and $\theta$. The critical divergence speed can be found from the minimal positive eigenvalue of problem (47).

For $T=0$ and $V_{0}=0$, from equation (40) we can formulate the problem of Guo and Païdoussis (2000) (see also Païdoussis 2004) in the limit of an infinitely wide channel:

$$
K f=\bar{U}^{2} N f
$$

where the eigenvalue $\bar{U}^{2}$ is the dimensionless dynamic pressure (Païdoussis 2004, p. 1149)

$$
\bar{U}^{2}=\frac{\rho_{f} \ell^{3}}{D} v_{\infty}^{2}
$$

which is the square of the dimensionless flow velocity $\bar{U}$. Equation (48) defines a standard linear generalised eigenvalue problem. The critical fluid flow velocity for divergence, $v_{\infty}^{\text {div }}$, can be found from ${\overline{U_{c d}}}^{2}$, the minimal positive eigenvalue $\bar{U}^{2}$ of the problem (48).

\section{Insert Figure 2 here}

Figure 2. Behaviour of $N(\xi, x)$ in $\Omega \equiv[-1,1] \times[-1,1]$. The infinities and the upper and left edges should be understood in the sense of limits.

Note that because the aerodynamic kernel $N(\xi, x)$ is singular along the line $x=\xi$, some care needs to be taken in computing the integrals in 
the matrix $N_{j n}$. We use the approximation

$$
\begin{aligned}
N_{j n} & \equiv \quad \int_{-1}^{1} \int_{-1}^{1} \frac{\mathrm{d} \Psi_{n}}{\mathrm{~d} x}(\xi) N(\xi, x) \frac{\mathrm{d} \Psi_{j}}{\mathrm{~d} x}(x) \mathrm{d} \xi \mathrm{d} x \\
& \approx \int_{-1+\varepsilon_{1}}^{1-\varepsilon_{1}}\left(\int_{-1}^{x-\varepsilon_{2}} \frac{\mathrm{d} \Psi_{n}}{\mathrm{~d} x}(\xi) N(\xi, x) \frac{\mathrm{d} \Psi_{j}}{\mathrm{~d} x}(x) \mathrm{d} \xi\right. \\
& \left.+\quad \int_{x+\varepsilon_{2}}^{1} \frac{\mathrm{d} \Psi_{n}}{\mathrm{~d} x}(\xi) N(\xi, x) \frac{\mathrm{d} \Psi_{j}}{\mathrm{~d} x}(x) \mathrm{d} \xi\right) \mathrm{d} x
\end{aligned}
$$

where $\varepsilon_{1}$ and $\varepsilon_{2}$ are small. It can be shown that the $L_{1}$ norm of $N(\xi, x)$ is finite. This property, together with the Hölder inequality guarantees that the integrals containing $N(\xi, x)$ converge. Thus, all $N_{j n}$ are finite and our approximation is legitimate.

Some useful properties may be obtained analytically for high-level optimisation of the numerical evaluation of $N_{j n}$. First, we note that the aerodynamic kernel $N(\xi, x)$ itself is symmetric along the straight lines $x=\xi$ and $x=-\xi$ inside the domain $(x, \xi) \in[-1,1] \times[-1,1]$. On the upper and left edges of the domain (see Figure 2) it is singular. However, if, at these locations, we instead understand the expression of $N(\xi, x)$ as a one-sided limit at the edge (approaching from inside the domain), then the symmetry applies there, too. Finally, $N(\xi, x)$ is undefined at exactly two corners of the domain, but this does not affect the integrals. These observations are summarised in Figure 2. The details for deriving all the properties stated in the last two paragraphs are given in Banichuk et al. (2008).

Then, considering the whole expressions of the integrals in $N_{j n}$, we make some more observations. If $j+n$ is odd, then $N_{j n}=0$ by consideration of symmetries of the integrand. The matrix is symmetric, by symmetry of $N(\xi, x)$ with respect to $x=\xi$, and application of Fubini's theorem. Finally, when $j+n$ is even, the integrand is symmetric with respect to $x=\xi$ and $x=-\xi$, so for each integral we only need to evaluate one half of the domain and then multiply the result by 2 .

For a concrete numerical estimation, let us assume an ideal membrane with $D=0$ and physical parameters typical for a paper web. As for its length, let us take $\ell=1 \mathrm{~m}$ as an example for the rest of this section.

It is numerically observed that antisymmetric vibration modes do not contribute to the critical divergence speed or shape, i.e., using either $n_{0}=$ $2 k+1$ or $n_{0}=2 k+2$ where $k=0,1, \ldots$ produces the same result. Let us set $n_{0}=55$ in the Galerkin series (37). The results for different fluid velocities $v_{\infty}$ are presented in Table 1.

The numerical solution approximates the solution of the continuous problem (represented by equation (24)) rather accurately. As an example, 
Table 1

Critical divergence speed of a membrane $(D=0)$ for different fluid velocities with the physical parameters $T=500 \mathrm{~N} / \mathrm{m}, m=80 \mathrm{~g} / \mathrm{m}^{2}$ and $\rho_{f}=1.25 \mathrm{~kg} / \mathrm{m}^{3}$. The length of the membrane is $\ell=1 \mathrm{~m}$.

\begin{tabular}{|c|c||c|c|}
\hline$v_{\infty}[\mathrm{m} / \mathrm{s}]$ & $V_{0 \text { mem }}^{\text {div }}[\mathrm{m} / \mathrm{s}]$ & $v_{\infty}[\mathrm{m} / \mathrm{s}]$ & $V_{0 \text { mem }}^{\text {div }}[\mathrm{m} / \mathrm{s}]$ \\
\hline \hline 0 & 30.6071 & 0 & 30.6071 \\
\hline 1 & 31.4552 & -1 & 29.7549 \\
\hline 10 & 38.8994 & -10 & 21.8972 \\
\hline 20 & 46.7651 & -20 & 12.7606 \\
\hline 30 & 54.1760 & -30 & 3.1692 \\
\hline 33 & 56.3032 & -33 & 0.1958 \\
\hline
\end{tabular}

Figure 3 shows the sensitivity of the solution $V_{0}^{\text {div }}$ on the number of terms $n_{0}$ in the Galerkin approximation when $v_{\infty}=0$ and physical parameters are as in Table 1.

\section{Insert Figure 3 here}

Figure 3. The sensitivity of the solution $V_{0}^{\text {div }}$ on the number of terms $n_{0}$ in the Galerkin approximation. Physical parameters are the same as in Table 1, and $v_{\infty}=0$. Note the logarithmic vertical axis in the second plot.

Beginning with the last known solution of the finite-dimensional problem, we obtain an approximation to the solution of the continuous problem by summing the difference of successive terms based on the curve fits, shown in Figure 3, up to a large value of $j$. The coefficients for the fits are calculated by solving $a j+b=f\left(v_{\text {diff }}(j)\right)$ from each fit expression, and performing a linear fit for the function $f$.

It was observed that the exponential fit converges faster, but fluctuates visibly above and below the data. The rational fit converges more slowly, but stays closer to the data. For obtaining the limit approximation for each, the upper limit of summation $j_{\max }=899$ was used for the exponential fit, and for the rational fit, $j_{\max }=5999$. The limit approximations from the two fits were found to be very close to each other.

With the physical parameters given above and $v_{\infty}=0$, we have from both fits the limit approximation $V_{0 \mathrm{mem}}^{\text {div }} \approx 30.6065 \mathrm{~m} / \mathrm{s}$. We notice that the added terms change the result by less than $0.002 \%$.

An important question is how the problem parameters influence the solution. In Figures 4 and 5, the effect of the parameters on the critical divergence speed can be seen. 
As the fluid velocity is increased, the critical divergence speed also becomes larger, until it suddenly drops to zero. This is because increasing $\theta$ shifts the whole spectrum of eigenvalues toward positive infinity. At some critical value of $\theta$ (depending on the other parameters), the maximal negative eigenvalue will cross the origin and become the minimal positive eigenvalue.

Because statical instability analysis is only concerned with the critical velocity, and not the (complex-valued) eigenfrequencies, the present analysis cannot tell apart the type of the interval between the origin $\left(V_{0}=0\right)$ and the minimal positive eigenvalue, i.e., whether the behaviour in the interval is stable or unstable. Of course, by physical considerations this interval is known to be stable when the fluid velocity is zero.

Due to this theoretical constraint, we have concentrated only on the lowest region of stability, given by the interval between the two eigenvalues that, at zero fluid velocity, are the maximal negative and the minimal positive eigenvalue. Once either end of this region crosses the origin (as $\theta$ is varied), the results become physically meaningless. The figures shown have been filtered to show only the physically meaningful data.

With this in mind, Figure 4 shows the effect of the fluid velocity $\theta$ (defined by (46)) and the bending parameter $\beta$ (defined by (44)) on the critical divergence speed in the lowest region of stability. The density ratio $\gamma$ has been fixed to the value 15.625 corresponding to the physical parameters used in Table 1. As expected, the region of stability expands toward materials having a larger bending stiffness $D$, or as the length of the span $\ell$ becomes shorter.

However, as the tension $T$ is increased, $\beta$ decreases. This is counterintuitive, as one would expect a stabilising effect resulting from increased tension. A stabilising effect is indeed present, because increasing the tension also increases the vacuum divergence velocity (equation (36)). This causes $\theta$ to decrease if the dimensional fluid velocity is kept constant, and at $\theta=0, \lambda$ stays constant with increasing $T$. Thus, one needs to be careful when interpreting the dimensionless results with respect to tension or paper density.

\section{Insert Figure 4 here}

Figure 4. Effect of fluid velocity on the critical divergence speed, parametrised by values of bending rigidity. The parameter $\gamma=15.625$.

\section{Insert Figure 5 here}

Figure 5. Effects of fluid density and fluid velocity on the critical divergence speed of a membrane, $D=0$. In the blank area, the eigenvalue problem has no physical solution.

The critical value of $\theta$, where the lowest region of stability ends, can be 
found for each fixed pair $(\beta, \gamma)$ numerically e.g. with a linear-logarithmic search procedure. That is, start from $\theta=0$, increase $\theta$ in fixed steps until the solution decreases, then go back one step, halve the step size and repeat the procedure until desired tolerance is achieved. For the cases illustrated in Figure 4, the values $\theta_{\text {critical }}$ are found to be 0.4199 for $\beta=0,0.8287$ for $\beta=1$, and 1.627 for $\beta=5$.

Figure 5 provides a closer look into the fluid parameters when $\beta=0$. The effect of the density ratio is plotted against the fluid velocity. The shading of each point in the figure represents the ratio of the critical divergence speed to the vacuum case. Light shades are the closest to the vacuum solution, and dark shades indicate that the critical divergence speed is much lower than the vacuum solution. The blank area lies outside the lowest stability region. The curve with $\beta=0$ in the previous Figure 4 shows a slice of this plot at the line $\gamma=15.625$.

In addition to the critical divergence speed, the corresponding divergence shapes are of interest. All of the shapes at different parameter values lie between two extremes. These are the vacuum shape, and the critical $\theta$ limit shape where the fluid exerts the largest effect. In the vacuum, the value of $\beta$ has no effect on the shape obtained. On the other hand, if there is no bending rigidity, any nonzero value of $\gamma$ causes the membrane to take the limit shape.

Figure 6 shows as an example the divergence shape at $\beta=0.01, \gamma=$ 15.625 and $\theta=\theta_{\text {critical }} \approx 0.43238$. The corresponding vacuum shape is also shown.

Figure 7 shows the difference between the vacuum shape and the shape at different $\theta$, with fixed $\gamma=15.625$ and two different values of $\beta$. The critical $\theta$ for $\beta=0.01$ was given in the previous paragraph. For $\beta=0.1$, we have $\theta_{\text {critical }} \approx 0.49161$.

The vertical scaling of Figure 7 corresponds to that in Figure 6. We observe that the fluid presses the ends of the shape down when compared to the vacuum case, whereas the center bulges slightly. The difference changes sign at $x \approx \pm 0.35$. We also observe that $\beta$ needs to be relatively small to obtain a noticeable difference from the vacuum divergence shape.

We can observe that for most pairs of the parameters $\beta$ and $\gamma$, the divergence shape is very close to one of its two extremes. That is, obtaining in-between shapes requires careful choice of the parameters. This behaviour is shown in Figure 8.

\section{Insert Figure 6 here}

Figure 6. Divergence shape at $\beta=0.01, \gamma=15.625, \theta=\theta_{\text {critical }} \approx 0.43238$ (solid line). The dashed line shows the corresponding vacuum divergence shape. 


\section{Insert Figure 7 here}

Figure 7. Difference between vacuum divergence shape and divergence shape for different fluid velocities at two different values of $\beta$. The parameter $\gamma=15.625$.

\section{Insert Figure 8 here}

Figure 8. Distance of the divergence shape from its extreme values. The parameter $k \equiv 201$. The axes have a logarithmic scale with base 10 .

The first two graphs in Figure 8 show the distance of the divergence shape from each of the extreme values. The upper graph represents the distance from the vacuum shape, and the lower graph represents the distance from the critical $\theta$ limit shape.

The third graph in Figure 8 shows the product of the two distances from the first two graphs. At parts of the graph where this function is large (in the relative sense), we have divergence shapes that are as far as possible from both extremes.

The sums in Figure 8 are calculated as follows. The Galerkin series (37) of each divergence shape $w$ is assembled with $k$ equally spaced points in the range $x \in[-1,1]$, and normalised by a constant such that the maximum displacement is +1 . Reference shapes are calculated for a membrane in vacuum $(\beta=0, \gamma=0)$, and for paper in air near critical $\theta(\beta=0$, $\gamma=15.625, \theta=\theta_{\text {critical }}-\varepsilon$ where $\varepsilon$ is small) and assembled as $w_{\text {vac }}$ and $w_{\text {crit }}$, respectively. The reference shapes are also normalised such that the maximum displacement is +1 .

Finally, let us compare our results to known ones. The study by Pramila (1986) is well suited for comparison, because in it an axially moving web submerged in ideal fluid was considered. In Pramila's study, we have $v_{\infty}=$ 0 for the stationary air, $D=0$ for the threadline model (corresponding to an ideal membrane in the flat panel model), and $\rho_{f}=1.2 \mathrm{~kg} / \mathrm{m}^{3}$.

In his study, Pramila gives the nondimensional first natural frequency as a function of the nondimensional velocity for some example cases. We are interested in the nondimensional velocity where the natural frequency becomes zero, denoted $\lambda$ in our eigenvalue problem.

From added mass considerations, Pramila obtains a scaling factor, $r$. In his model, the first natural frequency and the critical divergence velocity (computed from the vacuum case) both become scaled with the factor $r$, when the ideal fluid is taken into account. In our model, $\lambda$ is the scaling factor for the divergence velocity, and the present analysis makes no prediction concerning the natural frequency.

For $m=35.5 \mathrm{~g} / \mathrm{m}^{2}$, Pramila's Figure 5 (Pramila 1986, p. 74) suggests that $r$ is in the range $20 \%-30 \%$, depending on which added mass expression 
is used. Similarly, his Figure 6 suggests that for $m=54 \mathrm{~g} / \mathrm{m}^{2}, r$ is in the range $22 \%-35 \%$. For both these examples, the dimensions of the open draw are $2 \ell=2.4 \mathrm{~m}$ and $b=0.47 \mathrm{~m}$, leading to an aspect ratio of $2 \ell / b \approx 5.1$, i.e. a narrow strip. Note that we will not need the width in our model.

In both cases, we have the dimensionless parameters $\beta=0$ and $\theta=0$. Noting that for stationary fluid the value of $T$ only affects the absolute value of $V_{0}^{\text {div }}$ and not $\lambda$, our model predicts that for the first case $(\gamma=40.5634)$, $r \approx 25 \%$. For the second case $(\gamma=26.6667)$, we have $r \approx 31 \%$. Both cases show good agreement with Pramila's results.

Let us compare the results using another aspect ratio, too. In Pramila's numerical example (Pramila 1986, p. 72), the geometry is a wide plate with $2 \ell=0.75 \mathrm{~m}$ and $b=7.5 \mathrm{~m}$, giving an aspect ratio of 0.1 . The physical parameters are $T=16 \mathrm{~N} / \mathrm{m}$ and $m=50 \mathrm{~g} / \mathrm{m}^{2}$. This gives a vacuum divergence velocity of $V_{0}^{\mathrm{div}}$ vac $\approx 18 \mathrm{~m} / \mathrm{s}$. Pramila predicts that with these values, depending on the added mass expression used, the critical velocity is found to be between $2.7-4.6 \mathrm{~m} / \mathrm{s}$, or $15 \%-26 \%$ of the vacuum case.

Inserting the numbers to our model, we have $\beta=0, \gamma=9.0, \theta=0$, and solving the eigenvalue problem gives that $V_{0}^{\text {div }} \approx 8.7 \mathrm{~m} / \mathrm{s}$, or $48 \%$ of the vacuum case. Here the only agreement is qualitative: according to both models, the divergence velocity decreases when compared to the vacuum case. The quantitative difference is probably due to the deformation localisation effect, which renders the cylindrical deformation assumption invalid in the case of an axially moving wide plate.

As for the stationary plate subjected to axial flow, Païdoussis (2004) reports that (p. 1155) in a wide channel, the dimensionless critical flow velocity for a pinned-pinned plate is $\overline{U_{c d}} \approx 3.3$. By solving the eigenvalue problem (48), we obtain $\overline{U_{c d}} \approx 2.8$, which is within $20 \%$ of the referred value. We observe that the choice of $n_{0}$ does not matter; the change in the result between $n_{0}=1$ and $n_{0}=56$ is less than $0.5 \%$. The fast convergence agrees with the remark in Guo and Païdoussis (2000).

Païdoussis (2004) lists the quantity ${\overline{U_{c d}}}^{2} / \pi^{3}$ for several different references, where flow on one side only has been studied. Scaling $\overline{U_{c d}}$ by a factor of 2 to account for flow on one side only (Païdoussis 2004, p. 1155), we have for our result that $\left(2 \overline{U_{c d}}\right)^{2} / \pi^{3} \approx 0.99$. Comparing this to the results in the table on p. 1150 in the same reference (for number of antinodes $m=1$ ), we see that our result most closely corresponds to that of Dugundji et al. 1963 $\left({\overline{U_{c d}}}^{2} / \pi^{3}=1.00\right)$, as for the other results listed, the quantity is closer to 1.3. 


\section{Notes and conclusion}

In the previous sections, we considered the statical instability problem for travelling membranes and plates submerged in ideal fluid, when the elastic deformation is small and cylindrical. We discussed the case in which the external aerodynamic problem is two-dimensional, and the aerodynamic force is found analytically as an integral functional of the plate displacement function. As a result of analytical transformations, the original aeroelastic problem was reduced to the solution of an ordinary integrodifferential equation for the displacement function. To find the critical divergence speed, the obtained eigenvalue problem was solved numerically. The computations performed show that the surrounding fluid has a meaningful effect on the critical parameters of instability.

Furthermore, it was found that when working in an vacuum eigenmode basis, the aerodynamic coefficient matrix introduced due to the fluidstructure interaction is diagonally dominated, but not diagonal. Thus, when the plate is submerged in an external medium, even if this medium is ideal fluid, couplings exist between the vacuum eigenmodes that cannot be reproduced by an added mass approach. This conclusion is important for rigorous estimation.

For the most part of this study, the velocity of the moving plate was regarded as the unknown critical value characterising the statical form of instability i.e. divergence. Other essential values, such as tension, the densities of the fluid and plate, velocity of the fluid, and the bending rigidity of the plate, were considered as given. However, the problem can be easily restated to determine the critical values of these other parameters, as was briefly done for the critical velocity of the fluid in the special case of a stationary plate with no applied tension.

The model developed can, with some limiting assumptions, provide some insight into the divergence of a moving paper web interacting with air flowing along the axis of movement of the paper web. Taking this into account, we used for numerical estimations the parameter values corresponding to air and some paper materials.

It should be noted that the cylindrical deformation assumption is an approximation, due to the localisation of deformation near the free edges that has been observed in axially moving paper webs. Based on our comparison to earlier results (Pramila 1986), we conclude that the flat panel model is nevertheless a reasonable approximation for a narrow strip.

A recent study by Frondelius et al. (2006) suggests that due to the boundary layer, the divergence velocities in viscous fluid may be significantly higher than those predicted by the ideal fluid model. Thus, the present

results should be primarily seen as academical basic research concerning 
axial flow phenomena and axially moving materials.

Even though the model ignores the deformation localisation effect and fluid viscosity, much work still remains to be done even in the present simplified case, as the benefit of the approach was not fully realised in the statical analysis. The dynamical behaviour of the system, and dynamical instability analysis (Bolotin 1963), remain natural directions in which to extend this research.

Acknowledgement: This research was supported by the MASI Tekes Technology Programme. 


\section{A Derivation of aerodynamic reaction}

The purpose of this section is to present a derivation of the formulas (16)-(18) for the aerodynamic reaction.

To find $\varphi=\varphi(x, z)$, we consider the following linearised boundary value problem:

$$
\begin{gathered}
\triangle \varphi=\frac{\partial^{2} \varphi}{\partial x^{2}}+\frac{\partial^{2} \varphi}{\partial z^{2}}=0 \\
\left(\frac{\partial \varphi}{\partial z}\right)^{ \pm}=\gamma(x), \quad-\ell \leq x \leq \ell, z=0 \\
(\nabla \varphi)_{\infty}=0,
\end{gathered}
$$

where

$$
\gamma(x) \equiv\left(v_{\infty}-V_{0}\right) \frac{\mathrm{d} w}{\mathrm{~d} x} .
$$

We will solve this problem in the dimensionless notation (3)-(4).

We introduce an auxiliary function $W=\Psi+i \varphi$ of the complex variable $\zeta=x+i z$, where $i^{2}=-1$. The Cauchy-Riemann equations and the boundary conditions (A.2) imply that

$$
\frac{\partial \Psi}{\partial x}=\frac{\partial \varphi}{\partial z}=\gamma(x)
$$

and consequently we have

$$
\begin{gathered}
\Psi=\chi(x)+C \\
\chi(x)=\int_{-1}^{x} \gamma(\xi) \mathrm{d} \xi,
\end{gathered}
$$

where $C$ is a real constant of integration.

Thus, finding the potential is reduced to the computation of the imaginary part of the analytic function $W=\Psi+i \varphi$, whose real part on $[-1,1]$ is $\operatorname{Re} W=\Psi=\chi(x)+C$. We use the results given in Sherman (1952) and represent the solution to this problem as

$$
W(\zeta)=\frac{1}{2 \pi i}\left(\frac{\zeta-1}{\zeta+1}\right)^{1 / 2} \int_{-1}^{1}\left(\frac{\xi+1}{\xi-1}\right)^{1 / 2} \frac{\chi(\xi)+C}{\xi-\zeta} \mathrm{d} \xi .
$$

The real constant $C$ is determined with the help of the following equation,

$$
\frac{1}{2 \pi i} \int_{-1}^{1} \frac{\chi(\xi)+C}{\sqrt{\xi^{2}-1}} \mathrm{~d} \xi=0
$$


which represents a regularity condition for the function $W$ at the point $\zeta=$ 1. From the condition (A.9) we have

$$
C=\frac{1}{\pi i} \int_{-1}^{1} \frac{\chi(\xi) \mathrm{d} \xi}{\sqrt{\xi^{2}-1}}
$$

Using the expression (A.10) and the formula

$$
\frac{1}{2 \pi i} \int_{-1}^{1}\left(\frac{\xi+1}{\xi-1}\right)^{1 / 2} \frac{\mathrm{d} \xi}{\xi-\zeta}=\frac{1}{2}\left(\frac{\zeta+1}{\zeta-1}\right)^{1 / 2}-\frac{1}{2}
$$

we perform substitutions into expression (A.8) and elementary transformations, and obtain

$$
\begin{aligned}
W= & \frac{1}{2 \pi i}\left(\frac{\zeta-1}{\zeta+1}\right)^{1 / 2} \int_{-1}^{1}\left(\frac{\xi+1}{\xi-1}\right)^{1 / 2} \frac{\chi(\xi) \mathrm{d} \xi}{\zeta-\zeta} \\
+ & \frac{C}{2}\left[1-\left(\frac{\zeta-1}{\zeta+1}\right)^{1 / 2}\right]=\frac{\sqrt{\zeta^{2}-1}}{2 \pi i} \int_{-1}^{1} \frac{\chi(\xi) \mathrm{d} \xi}{(\xi-\zeta) \sqrt{\xi^{2}-1}}+\frac{C}{2} .
\end{aligned}
$$

To compute the quantity $\varphi^{+}$, we take the limit of (A.12) at $\zeta=x+$ $i z \rightarrow x+i \cdot 0\left(z \rightarrow 0^{+}\right)$and separate the imaginary part

$$
\begin{aligned}
\varphi^{+} & =\lim _{z \rightarrow 0^{+}}[\operatorname{Im} W(x+i z)] \\
& =\quad \text { p.v. }\left(-\frac{\sqrt{1-x^{2}}}{2 \pi} \int_{-1}^{1} \frac{\chi(\xi) \mathrm{d} \xi}{(\xi-x) \sqrt{1-\xi^{2}}}\right) .
\end{aligned}
$$

Here we took into account that the constant $C$ on the right-hand side of (A.12) is real, and consequently must be omitted when the limit of the imaginary part is computed in (A.13). Note also that the integration in (A.13) is understood in the sense of Cauchy's principal value (p.v.).

Thus we have

$$
\varphi^{+}-\varphi^{-}=2 \varphi^{+}=\text {p.v. }\left(-\frac{\sqrt{1-x^{2}}}{\pi} \int_{-1}^{1} \frac{\chi(\xi) \mathrm{d} \xi}{(\xi-x) \sqrt{1-\xi^{2}}}\right) .
$$


By definition of the principal value, we will have

$$
\begin{aligned}
2 \varphi^{+}=\text {p.v. } & \left(\frac{1}{\pi} \int_{-1}^{1}\left(\frac{1-x^{2}}{1-\xi^{2}}\right)^{1 / 2} \frac{\chi(\xi) \mathrm{d} \xi}{\xi-x}\right) \\
=\lim _{\varepsilon \rightarrow 0} & \frac{1}{\pi}\left[\int_{-1}^{x-\varepsilon}\left(\frac{1-x^{2}}{1-\xi^{2}}\right)^{1 / 2} \frac{\chi(\xi) \mathrm{d} \xi}{\xi-x}\right. \\
& \left.+\int_{x+\varepsilon}^{1}\left(\frac{1-x^{2}}{1-\xi^{2}}\right)^{1 / 2} \frac{\chi(\xi) \mathrm{d} \xi}{\xi-x}\right] .
\end{aligned}
$$
we have

Integrating by parts and substituting the expression (A.7) for $\chi(x)$,

$$
\begin{array}{r}
\lim _{\epsilon \rightarrow 0}\left[N(x-\epsilon, x) \int_{-1}^{x-\epsilon} \gamma(\xi) \mathrm{d} \xi-N(x+\epsilon, x) \int_{-1}^{x+\epsilon} \gamma(\xi) \mathrm{d} \xi\right. \\
\left.-\int_{-1}^{x-\epsilon} N(\xi, x) \gamma(\xi) \mathrm{d} \xi-\int_{x+\epsilon}^{1} N(\xi, x) \gamma(\xi) \mathrm{d} \xi\right]
\end{array}
$$

where we use the notation

$$
N(\xi, x) \equiv \frac{1}{\pi} \ln \left|\frac{1+\Lambda}{1-\Lambda}\right|, \quad \Lambda(\xi, x) \equiv\left[\frac{(1-x)(1+\xi)}{(1-\xi)(1+x)}\right]^{1 / 2} .
$$

We observe that all terms on the right-hand side of (A.16) are finite; therefore, the integration by parts is legitimate. As $\varepsilon \rightarrow 0$, the sum of the first two terms in (A.16) approaches zero, while the last two integrals converge. Therefore, the required functional dependence is of the form

$$
2 \varphi^{+}=-\int_{-1}^{1} N(\xi, x) \gamma(\xi) \mathrm{d} \xi
$$

With the help of (10), (A.4), (A.17), and (A.18), we derive the expression for the aerodynamic reaction of the fluid:

$$
\begin{aligned}
& q_{f}(x)=\quad p^{-}(x)-p^{+}(x)=\quad \rho_{f} \mathcal{v}\left[\left(\frac{\partial \varphi}{\partial x}\right)^{+}-\left(\frac{\partial \varphi}{\partial x}\right)^{-}\right] \\
& =\quad \rho_{f} v \frac{\partial}{\partial x}\left[\varphi^{+}-\varphi^{-}\right]=\quad \rho_{f} v \frac{\partial}{\partial x}\left[2 \varphi^{+}\right] \\
& =-\rho_{f} v \frac{\partial}{\partial x} \int_{-1}^{1} N(\xi, x) \gamma(x) \mathrm{d} \xi=-\rho_{f} v^{2} \frac{\partial}{\partial x} \int_{-1}^{1} N(\xi, x) \frac{\mathrm{d} w}{\mathrm{~d} x}(\xi) \mathrm{d} \xi,
\end{aligned}
$$

where we have abbreviated $v \equiv v_{\infty}-V_{0}$. 


\section{References}

Archibald, F. R., Emslie, A. G., 1958. The vibration of a string having a uniform motion along its length. ASME Journal of Applied Mechanics 25, 347-348.

Banichuk, N., Jeronen, J., Neittaanmäki, P., Tuovinen, T., 2008. Instability analysis for traveling membranes and plates interacting with surrounding ideal fluid. Tech. Rep. 13, University of Jyväskylä.

Banichuk, N., Neittaanmäki, P., 2008. On the transverse vibrations analysis of traveling membranes and plates submerged into ideal fluid. Tech. Rep. 2, University of Jyväskylä.

Bisplinghoff, R. L., Ashley, H., 1962. Principles of Aeroelasticity. Dover Publ. Inc., New York, 2nd edition 1975.

Bolotin, V. V., 1963. Nonconservative Problems of the Theory of Elastic Stability. Pergamon Press, New York.

Chang, Y. B., Moretti, P. M., 1991. Interaction of fluttering webs with surrounding air. TAPPI journal.

Chonan, S., 1986. Steady state response of an axially moving strip subjected to a stationary lateral load. J. Sound Vib. 107, 155-165.

Dugundji, J., Dowell, E., Perkin, B., 1963. Subsonic flutter of panels on continuous elastic foundations. AIAA Journal 1 (5), 1146-1154.

Eloy, C., Souilliez, C., Schouveiler, L., 2007. Flutter of a rectangular plate. J. Fluids Struct. 23 (6), 904-919.

Frondelius, T., Koivurova, H., Pramila, A., 2006. Interaction of an axially moving band and surrounding fluid by boundary layer theory. J. Fluids Struct. 22 (8), 1047-1056.

Guo, C. Q., Païdoussis, M. P., 2000. Stability of rectangular plates with free side-edges in two-dimensional inviscid channel flow. ASME J Appl Mech $67,171-176$.

Huang, L., 1995. Flutter of cantilevered plates in axial flow. J. Fluids Struct. 9 (2), 127-147.

Kornecki, A., Dowell, E. H., O’Brien, J., 1976. On the aeroelastic instability of two-dimensional panels in uniform incompressible flow. Journal of Sound and Vibration 47 (2), 163-178.

Kulachenko, A., Gradin, P., Koivurova, H., 2007a. Modelling the dynamical behaviour of a paper web. part i. Computers \& Structures 85, 131-147.

Kulachenko, A., Gradin, P., Koivurova, H., 2007b. Modelling the dynamical behaviour of a paper web. part ii. Computers \& Structures 85, 148-157.

Miranker, W. L., 1960. The wave equation in a medium in motion. IBM J. R\&D 4, 36-42.

Mujumdar, A. S., Douglas, W. J. M., 1976. Analytical modelling of sheet flutter. Svensk Papperstidning 79, 187-192.

Niemi, J., Pramila, A., 1986. Vibration analysis of an axially moving membrane immersed into ideal fluid by fem. Tech. rep., Tampere.

Païdoussis, M. P., 2004. Fluid-Structure Interactions: Slender Structures and 
Axial Flow. Vol. 2. Elsevier Academic Press.

Païdoussis, M. P., 2008. The canonical problem of the fluid-conveying pipe and radiation of the knowledge gained to other dynamics problems across applied mechanics. Journal of Sound and Vibration 310, 462-492.

Pramila, A., 1986. Sheet flutter and the interaction between sheet and air. TAPPI-Journal 69 (7), 70-74.

Pramila, A., 1987. Natural frequencies of a submerged axially moving band. Journal of Sound and Vibration 113 (1), 198-203.

Sedov, L. I., 1972. A Course in Continuum Mechanics, english Edition. Vol. 3. Wolters-Noordhoff Publishing, Groningen, Netherlands, chapter 9: Potential motion.

Shen, J. Y., Sharpe, L., McGinley, W. M., 0 1995. Identification of dynamic properties of plate-like structures by using a continuum model. Mechanics Research Communications, 22 (1), 67-78.

Sherman, D. I., 1952. On the stress distribution in partitions, an elastic heavy medium which is weakened by elliptic holes. Izv. Akad. Nauk SSSR, OTN 7, 992-1010.

Shin, C., Chung, J., Kim, W., 9/22 2005. Dynamic characteristics of the outof-plane vibration for an axially moving membrane. Journal of Sound and Vibration, 286 (4-5), 1019-1031.

Simpson, A., 1973. Transverse modes and frequencies of beams translating between fixed end supports. J. Mech. Eng. Sci. 15, 159-164.

Swope, R. D., Ames, W. F., 1963. Vibrations of a moving threadline. J. Franklin Inst. 275, 36-55.

Sygulski, R., 1 2007. Stability of membrane in low subsonic flow. International Journal of Non-Linear Mechanics, 42 (1), 196-202.

Timoshenko, S. P., Woinowsky-Krieger, S., 1959. Theory of plates and shells, 2nd Edition. New York: Tokyo : McGraw-Hill.

Tisseur, F., Meerbergen, K., 2001. The quadratic eigenvalue problem. SIAM Rev. 43, 235-286.

Wang, X., 1 2003. Instability analysis of some fluid-structure interaction problems. Computers \& Fluids, 32 (1), 121-138.

Watanabe, Y., Isogai, K., Suzuki, S., Sugihara, M., 2002. A theoretical study of paper flutter. J. Fluids Struct. 16 (4), 543-560.

Wickert, J. A., Mote, C. D., 1990. Classical vibration analysis of axially moving continua. J. Appl. Mech. 57, 738-744.

$\mathrm{Wu}, \mathrm{X} ., \mathrm{Kaneko}$ S., 2005. Linear and nonlinear analyses of sheet flutter induced by leakage flow. J. Fluids Struct. 20 (7), 927-948. 


\section{Figure captions}

Figure 1: Travelling plate, supported at $x=-\ell$ and $x=+\ell$, buckling when its velocity is $V_{0}=V_{0}^{\text {div }}$. The displacement $w$ of the plate is assumed to be cylindrical, i.e., constant in the $y$ direction (perpendicular to the page, not shown). At infinity, the fluid moves along the $x$ axis with the velocity $v_{\infty}$. The plate loses its stability at some critical divergence velocity $V_{0}^{\text {div }}$.

Figure 2: Behaviour of $N(\xi, x)$ in $\Omega \equiv[-1,1] \times[-1,1]$. The infinities and the upper and left edges should be understood in the sense of limits.

Figure 3: The sensitivity of the solution $V_{0}^{\text {div }}$ on the number of terms $n_{0}$ in the Galerkin approximation. Physical parameters are the same as in Table 1 , and $v_{\infty}=0$. Note the logarithmic vertical axis in the second plot.

Figure 4: Effect of fluid velocity on the critical divergence speed, parametrised by values of bending rigidity. The parameter $\gamma=15.625$.

Figure 5: Effects of fluid density and fluid velocity on the critical divergence speed of a membrane, $D=0$. In the blank area, the eigenvalue problem has no physical solution.

Figure 6: Divergence shape at $\beta=0.01, \gamma=15.625, \theta=\theta_{\text {critical }} \approx$ 0.43238 (solid line). The dashed line shows the corresponding vacuum divergence shape.

Figure 7: Difference between vacuum divergence shape and divergence shape for different fluid velocities at two different values of $\beta$. The parameter $\gamma=15.625$.

Figure 8: Distance of the divergence shape from its extreme values. The parameter $k \equiv 201$. The axes have a logarithmic scale with base 10 . 


\section{Tables}

Table 1

Critical divergence speed of a membrane $(D=0)$ for different fluid velocities with the physical parameters $T=500 \mathrm{~N} / \mathrm{m}, m=80 \mathrm{~g} / \mathrm{m}^{2}$ and $\rho_{f}=1.25 \mathrm{~kg} / \mathrm{m}^{3}$. The length of the membrane is $\ell=1 \mathrm{~m}$.

\begin{tabular}{|c|c||c|c|}
\hline$v_{\infty}[\mathrm{m} / \mathrm{s}]$ & $V_{0 \text { mem }}^{\text {div }}[\mathrm{m} / \mathrm{s}]$ & $v_{\infty}[\mathrm{m} / \mathrm{s}]$ & $V_{0 \text { mem }}^{\text {div }}[\mathrm{m} / \mathrm{s}]$ \\
\hline \hline 0 & 30.6071 & 0 & 30.6071 \\
\hline 1 & 31.4552 & -1 & 29.7549 \\
\hline 10 & 38.8994 & -10 & 21.8972 \\
\hline 20 & 46.7651 & -20 & 12.7606 \\
\hline 30 & 54.1760 & -30 & 3.1692 \\
\hline 33 & 56.3032 & -33 & 0.1958 \\
\hline
\end{tabular}


Surrounding medium (air)

$$
\mathrm{x}=-\ell
$$

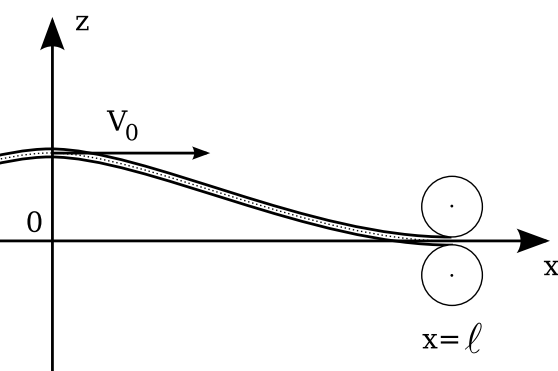

Surrounding medium (air) 


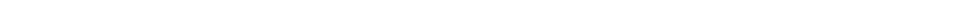


Figure $3 \mathrm{~V}_{0}^{\text {div }}$ vs. number of terms in approximation; $\mathrm{V}_{0}^{\text {div }}(55)=30.607143857 \mathrm{~m} / \mathrm{s}$
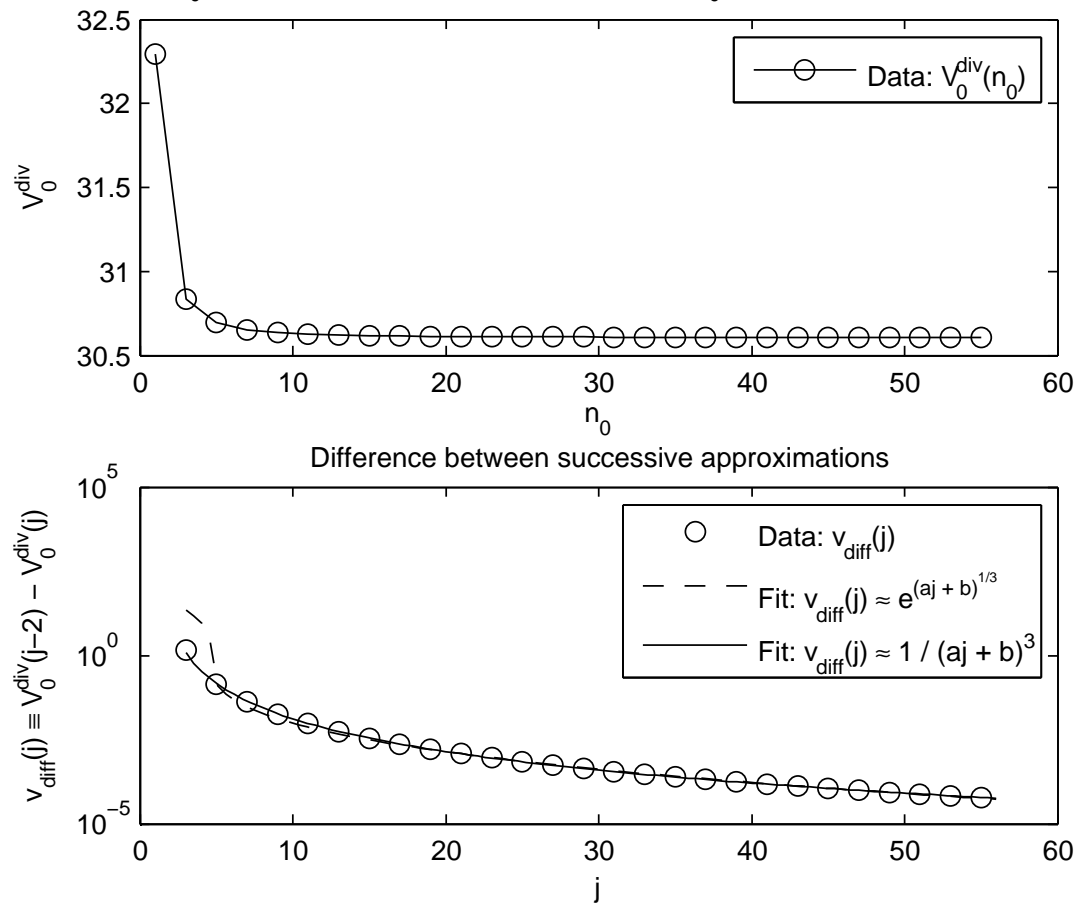
Figutye 4

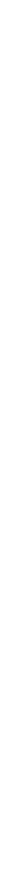


Figure 5

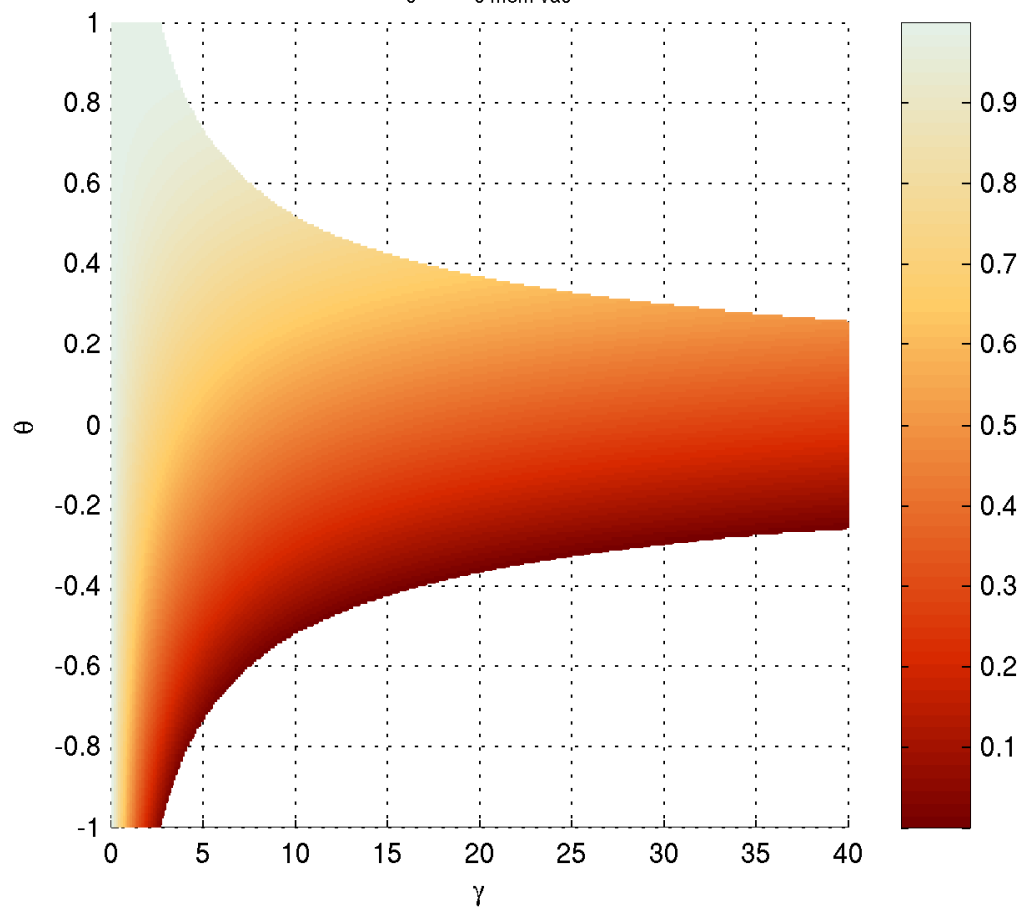


Figure 6

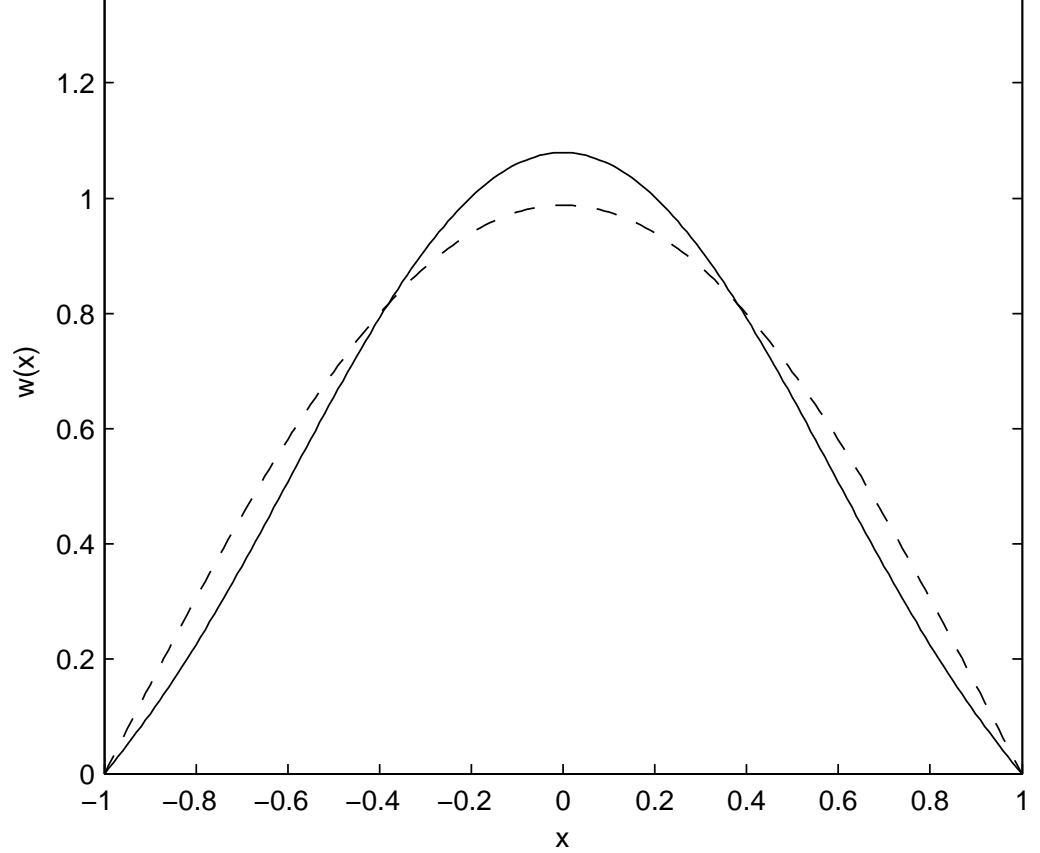


Figure 7

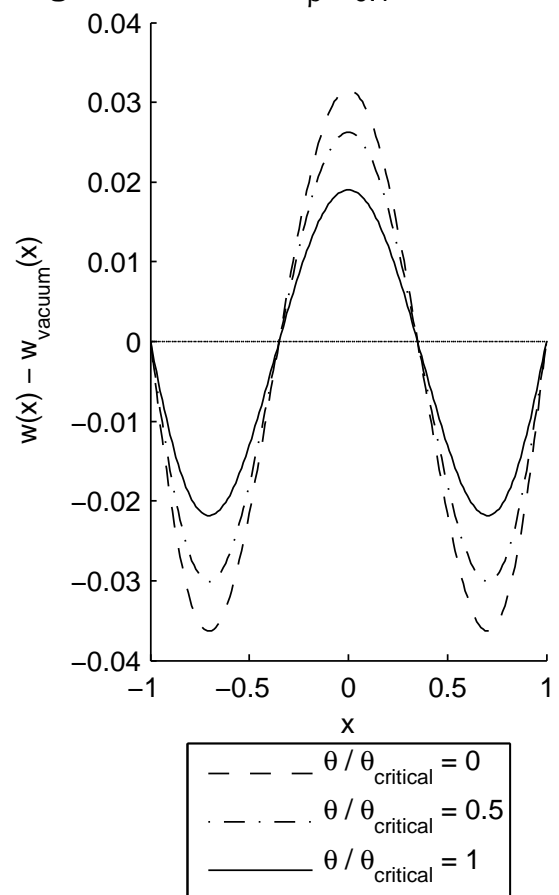

$\beta=0.01$

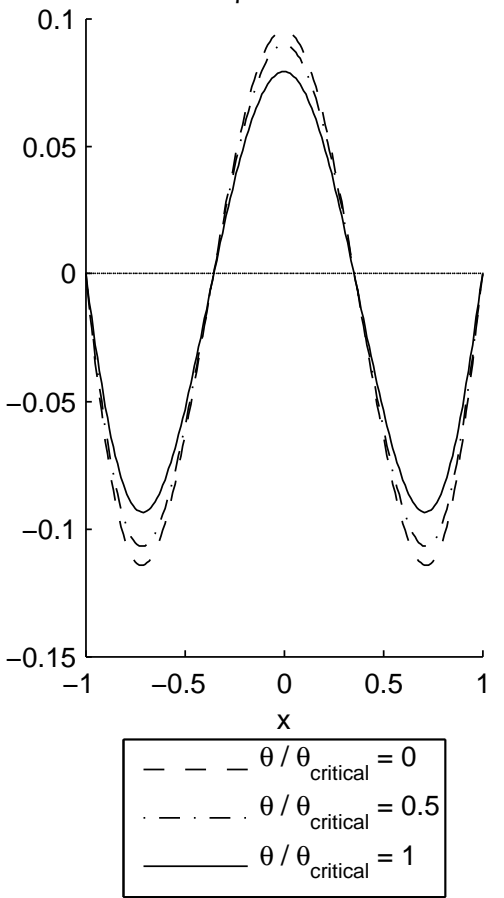


